\title{
Better Understanding of Factors Influencing Likelihood of Using Shared Bicycle Systems and Frequency of Use
}

\author{
Julie Bachand-Marleau, Brian H. Y. Lee, and Ahmed M. El-Geneidy
}

\begin{abstract}
Planning and transportation professionals are promoting a variety of sustainable travel alternatives, such as public transit usage, walking, and cycling, as affordable transportation options to counter the negative effects of widespread car use. In their traditional form, these alternative transport modes do not always offer the flexibility or convenience of the car; therefore, innovative solutions have been developed to allow active and public transport to compete better with the car. Shared bicycle systems have been adopted by a growing number of cities and regions throughout the world, yet little is known about the users of the systems and their motivations. A survey was conducted in Montreal, Quebec, Canada, in the summer of 2010 to determine the factors that encouraged individuals to use the system and the elements that influenced frequency of use. The factor found to have the greatest effect on the likelihood for use of a shared bicycle system was the proximity of home to docking stations. Ownership of a yearly shared bicycle membership was associated with cyclists riding shared bicycles 15 additional times per year. Respondents indicated that they valued the shared bicycle's trendy status and the role that it could play in bicycle theft prevention. The potential of shared bicycle systems can be maximized by increasing the number of docking stations in residential neighborhoods and by emphasizing the popularity of shared bicycles and theft prevention in advertising campaigns.
\end{abstract}

Bicycle sharing systems are a promising initiative for encouraging cycling, whose benefits to the user and to society as a whole are well known. Cycling is a form of physical activity in which health authorities place great hope because it can be easily incorporated into daily routines and yields cardiovascular benefits for both children and adults (1). It is also an environmentally friendly transportation mode that provides additional mobility at an affordable cost (2).

Bicycle sharing systems are intended to yield the benefits associated with cycling while providing additional convenience for the user, with the goal of convincing more people to adopt the bicycle for short trips. A bicycle sharing program enables individuals to use bicycles

J. Bachand-Marleau, Networks Development Group, AMT, 25th Floor, 500 Place d'Armes, Montreal, Quebec H2Y 2W2, Canada. B. H. Y. Lee, School of Engineering, Transportation Research Center, University of Vermont, 114 Farrell Hall, 210 Colchester Avenue, Burlington, VT 05405-0303. A. M. El-Geneidy, School of Urban Planning, McGill University, Suite 400, 815 Sherbrooke Street West, Montreal, Quebec H3A 2K6, Canada. Corresponding author: A. M. El-Geneidy, ahmed.elgeneidy@mcgill.ca.

Transportation Research Record: Journal of the Transportation Research Board, No. 2314, Transportation Research Board of the National Academies, Washington, D.C., 2012, pp. 66-71.

DOI: 10.3141/2314-09 whenever needed and without most of the costs and responsibilities associated with owning a bicycle (2). The flexibility of this transport mode makes it especially suitable for short distances and for one-way trips. All these characteristics have prompted a growing number of cities to implement bicycle sharing programs, which are present in more than 125 cities on four continents, about 140,000 shared bicycles worldwide (2).

Despite the growing popularity of shared bicycle systems, little is known about users of shared bicycles, about their reasons for using this form of transportation, and about the demand for shared bicycle programs. Research was done to determine the socioeconomic and spatial factors that influence someone's likelihood of using shared bicycles. This paper looks at those who are already using the bicycle sharing system and analyzes characteristics influencing their frequency of use. The research is based on a detailed online survey conducted in Montreal, Quebec, Canada, in the summer of 2010. The survey included demographic, travel behavior, and spatial questions to determine the elements affecting the use of and opportunities for cycle-transit integration and contained a section on the use of BIXI, Montreal's shared bicycle system.

\section{LITERATURE}

Bicycle sharing is a relatively recent concept; the first large-scale system was implemented in the Netherlands less than 50 years ago, in 1965. Bicycle sharing systems went through four major phases. The first generation, "white bikes," consisted of unlocked bicycles randomly located throughout the city. The bicycles were painted in one bright color. They could be picked up and left anywhere in the city, and their use was free of charge. In most cases, including in Amsterdam; Cambridge, United Kingdom; and Milan, Italy, the programs were put to an end after a few years because of the high number of damaged or stolen bicycles (2-4).

A second generation of bicycle sharing systems, the coin-deposit system, was introduced in the 1990s to overcome the problems encountered with the first-generation programs. These systems were characterized by the unique, robust design and bright color of the bicycles as well as designated docking stations where bicycles were borrowed and returned (3). The stations were equipped with a locking system to minimize theft risk, and borrowing a bicycle required a small deposit that was generally refunded on return. Although an improvement on the previous generation, the coin-deposit system did not completely solve the theft problem because of the anonymity of the borrowing process. Furthermore there was no time limit to bicycle usage, which caused people to borrow bicycles for unduly long periods (2). Most 
of these systems were implemented in Northern European countries, such as Denmark and the Netherlands (4).

The third-generation systems kept some of the second generation's features, such as the distinctive design of the bicycles and the presence of docking stations. In addition, these bicycle sharing programs incorporated transaction kiosks that allowed for identification of users (with portable phone or credit card number). These systems succeeded in reducing theft rates because users were subject to penalties if they failed to return the bicycles to a station (2). Users also had to have a membership to use the service. Typically, use was free for a certain period of time (in most cases, from half an hour to an hour), and then users were charged for the extra minutes, which encouraged shorter trips. The first city to implement such a system was Lyon, France, in 2005; many other European cities soon followed $(2,5)$.

The latest and fourth generation of bicycle sharing programs, the demand-responsive multimodal system, consists of management and efficiency improvements to the third-generation systems. The innovations include mobile or solar-powered docking stations, the use of smartcards, and bicycle distribution systems (2, 3, 6). Distribution systems involve moving shared bicycles from one station to another to ensure that bicycles and empty racks are always available for users to borrow and return a bicycle at any station. The shared bicycle system of Montreal is included in this last category of shared bicycle systems.

The literature on the evolution of bicycle sharing systems is limited but reliable and relatively easy to access. However, few research studies have explored the characteristics of users of bicycle sharing systems and the motivators for or deterrents to the use of shared bicycles. Little is known about the influence of socioeconomic, spatial, or behavioral characteristics of users of sharing systems or about the attributes of the system itself, such as pricing, extent of the network, availability of bicycles, or location of docking stations.

\section{CASE STUDY}

\section{Cycling and Bicycle Sharing in Montreal}

According to the latest Origine-Destination survey (a regional transportation survey that takes place every 5 years), the modal share of cycling in the region of Montreal is around 1.2\% of all trips (7). In the past few years, the city of Montreal expressed a commitment to improve cycling conditions. The transportation plan of the city launched in 2008 specified many interventions to reach that goal, such as implementing the BIXI system, doubling the cycling network, and increasing the number of bicycle racks for parking fivefold. Although the plan is not yet complete, the size of the network has increased steadily since its implementation. However, bicycle theft is a problem in Montreal. According to the city's police department, about 2,500 bicycles are reported stolen every year on average, yet this number represents only a small proportion of all bicycle thefts (A. Tremblay and N. Letendre, personal communication, 2011).

\section{BIXI, Montreal's Bicycle Sharing System}

The bicycle sharing system of Montreal, BIXI (a contraction of bicycle and taxi), was launched in the spring of 2009. It was one of the first demand-responsive multimodal systems to be implemented.
The BIXI system is in operation from April 15 to November. At the time BIXI was launched, about 300 stations were available, but the instant success of the system prompted the BIXI organization to implement the expansion phase ahead of schedule. There are currently about 405 stations throughout the central neighborhoods of Montreal, and a total of 5,050 bicycles are in circulation (8). The BIXIs were designed specifically for shared use in an urban context: they are robust yet aesthetically pleasant and are convenient for users, with their adjustable seats, front racks, and integrated chain protector, which keeps chain grease off riders' clothing (9). There are three BIXI membership types: the 24-h pass, the monthly membership, and the yearly membership. The system is meant to accommodate short trips, with the first half hour of use free and a charge for additional time. Discounts are available for individuals who combine their BIXI membership with an annual transit pass or a membership for Communauto (the carsharing service in Montreal). As of the fall of 2010, more than 3,000,000 trips have been made by BIXI (10).

\section{DATA}

An online survey about cycling and transit integration was conducted in the Montreal region in the summer of 2010. The questionnaire consisted of six sections: general travel habits, transit questions, cycling-transit questions, priorities for improving cycling and transit integration, general demographics, and comments section. It also included a separate section on BIXI usage, one of the viable options for integrating cycling and public transit. An uncontrolled online distribution method was used for the survey; anyone could go to the web page and fill out the questionnaire, which was available online for approximately 1 month. Many media, such as mailing lists, e-mail newsletters, social networking media, radio and newspaper interviews, and flyers distributed at major transit stations, were used to publicize the survey to ensure that a large cross section of the general population would be reached. The use of such a variety of means allowed for broader exposure, minimizing the bias associated with online surveys (11).

A total of 1,787 responses to the survey were gathered; after the incomplete observations were removed, a final sample of 1,432 respondents was obtained. Respondent age ranged from 18 to 87; however, the majority were between the ages of 25 and 35. Men were slightly more numerous than were women in the sample and accounted for $58 \%$ of the respondents. Young people with no children were overrepresented among the respondents compared with the Montreal population, the majority of these respondents living in small households of 1 or 2 people. More detailed descriptive statistics of the respondents are presented in Table 1.

Individuals who participated in the survey lived on average $6 \mathrm{~km}$ from downtown Montreal, and they had good access to transit with an average of 12 bus stops within $400 \mathrm{~m}$ of their residence. This may explain why the majority of respondents were bus users (took the bus at least once in the past year). Respondents also enjoyed good access to the shared bicycle system, with almost $60 \%$ living close to a BIXI station. About $87 \%$ of people in the sample had a valid driver's license, and 52\% owned at least one car per household.

One section of the survey specifically asked about the cycling habits of respondents. Almost all (94\%) owned a bicycle. Close to $40 \%$ of the respondents indicated they had had one bicycle stolen, and $10 \%$ had had two or more bicycles stolen. Almost $17 \%$ of the survey participants used their bicycles for transportation year round, 
TABLE 1 Selected Descriptive Statistics of Respondents

\begin{tabular}{lr}
\hline Statistic & Average \\
\hline Age (years) & 35.7 \\
Home-to-downtown distance (km) & 6.0 \\
Number of bus stops within 400 m of home & 12.1 \\
Number of bicycles stolen per respondent & 0.7 \\
Gender (female) (\%) & 41.8 \\
Recreational cyclists only (\%) & 6.6 \\
Year-round cyclists (\%) & 16.7 \\
Bus user (\%) & 76.8 \\
Yearly BIXI membership (\%) & 16.7 \\
People living within 500 m of a BIXI station (\%) & 59.4 \\
Respondents who had a bicycle stolen (\%) & 39.0 \\
\hline
\end{tabular}

and another $6 \%$ cycled only for recreational purposes. Thirty-seven percent of respondents indicated they had already used BIXI. For this group of BIXI users, membership types were split almost evenly between pay-per-use and yearly enrollments, and only $1 \%$ of BIXI users had acquired a monthly pass. Respondents were also asked about their motivations for using BIXI. These motivations and their answers to more specific questions concerning how they integrated BIXI into their transportation habits are presented in Figure 1.

Figure 1 shows that respondents used BIXIs for a variety of reasons, mostly for convenience or to avoid maintenance and risk of theft. Most people in the sample used BIXI for trips they previously made by transit or with their own bicycle. Only $10 \%$ of the respondents indicated that they used a BIXI instead of taking their car or a taxi; the environmental benefits of BIXI therefore are probably not the main advantage of the system. Despite BIXI's potential for multimodal trips, acknowledged by the respondents themselves, less than half the users combined BIXI with another mode for a trip.

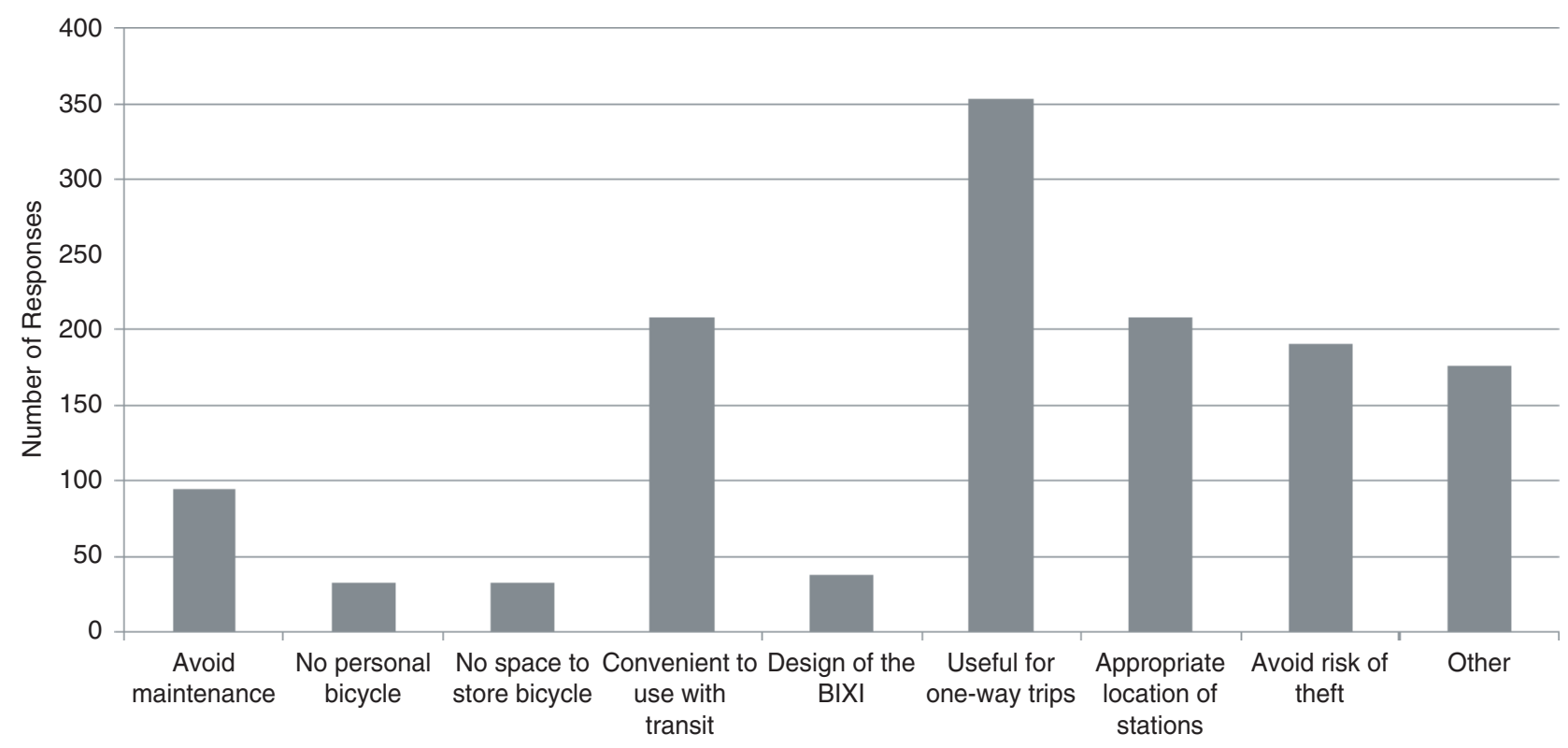

Answers

(a)

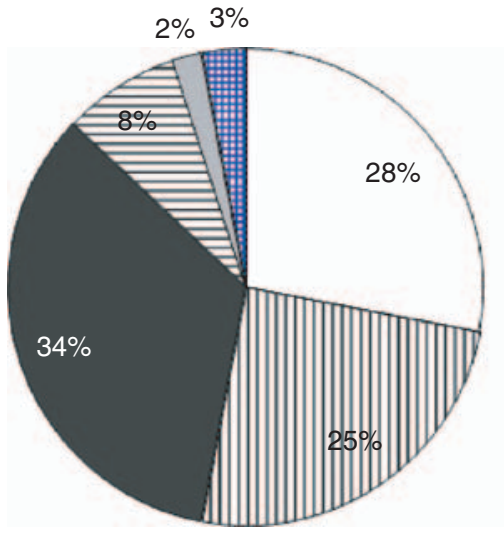

(b)

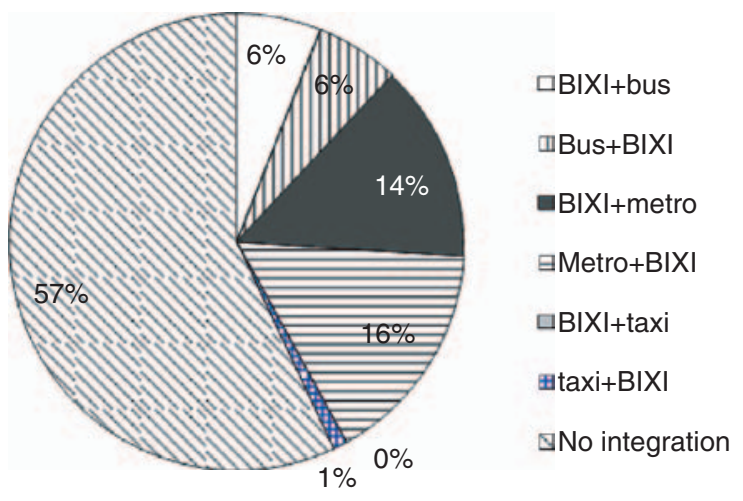

(c)

FIGURE 1 BIXI use by respondents: (a) reasons for use, $(b)$ travel modes replaced by BIXI, and $(c)$ integration of BIXI with other travel modes. 
When they did so, most respondents used BIXI in combination with the metro.

\section{METHODOLOGY}

Two types of regressions were used to answer the research questions. First, a binary logistic model was developed to determine factors encouraging the use of BIXI. A binary logistic model is a type of logistic regression in which the dependant variable is binary. In this case, the dependant variable is the previous use of a BIXI (yes-no). Then, a linear regression was applied on our subsample of BIXI users to identify factors that affect the frequency of use of shared bicycles. The dependant variable in this second model is the number of BIXI uses for the 2010 season. The first model tested a variety of variables based on results of previous studies that examined motivators for cycling in general or use of shared bicycle systems specifically. According to those studies, the typical user of a shared bicycle system is a young man earning a middle-class income (12). The analysis in this study was hypothesized to confirm that trend, and thus age, gender, and income were included as variables in the model.

It was also expected that travel habits would affect the likelihood of BIXI use; it was hypothesized that people who cycle only for recreation would be less likely to take part in a bicycle sharing system. It was supposed that owning a bicycle and being a committed, year-round cyclist would decrease the probability a person would use BIXI. Shared bicycle systems allow combining cycling and transit trips or replacing short bus trips; therefore, it was expected that bus users and people who already combined cycling and transit for a trip would be more likely to use shared bicycles. In contrast, it was hypothesized that having a driver's license would decrease one's odds of being a BIXI user.

A study of BIXI by Morency et al. identified proximity to docking stations as an important motivator for use of the system (6). The analysis in this study was expected to generate similar results, especially because that earlier study focused specifically on shared bicycles in Montreal. Living close to downtown was also expected to increase one's likelihood of using BIXI, because this residential location is associated with shorter, more bikeable travel distances. Finally, a hypothesis not yet tested in the literature and specifically related to Montreal's cycling context was explored. Bicycle theft is a problem in Montreal, and fear of theft can deter people from using their bicycles for transportation. Shared bicycles are a good alternative because users do not own the bicycles and therefore do not need to worry about a bicycle being stolen while it is parked. Consequently, it was expected that those whose bicycles have been stolen and thus are more conscious of theft risk would be more likely to use shared bicycles.

Many of the same variables were included in the second model, yet other distinct factors could also influence the frequency of use. First, the model incorporated a type of shared bicycle membership; it was expected that owning a yearly membership with unlimited access to the BIXI system for the first half hour would encourage people to use the system more often. Variables were added representing reasons for using shared bicycles to determine if some of the advantages of BIXI affect user behavior. Avoiding maintenance, avoiding risk of theft, and liking the design of BIXI (which can be considered as the "trendiness" factor of the BIXI) were among the most popular reasons for use mentioned by respondents and therefore were included in the model. Finally, the number of bus stops within a 400-m buffer of a residential location was added as a variable representing the level of transit access for respondents. Good access to transit was expected to diminish the need for shared bicycles. All variables included in both models were tested for correlation. Other variables, such as household size, car ownership, and a variety of interaction variables, were tested in the two regressions but proved to be insignificant and were therefore removed from the final models. The two models presented here are those that could explain the highest proportion of variance among the data with meaningful and significant variables.

\section{ANALYSIS}

Table 2 shows the results of the binary logistic regression that measured the probability of use of BIXI during the 2010 season. Three main types of variables were shown to have a significant role in the likelihood for use of shared bicycle systems: socioeconomic characteristics, transportation habits, and spatial characteristics. The variable with the strongest impact was the presence of a BIXI station less than $500 \mathrm{~m}$ from home, which made an individual more than $300 \%$ more likely to use a shared bicycle and confirmed the results of previous studies that stressed the importance of proximity to docking stations at the home location (8). The proximity of a BIXI station to a respondent's most regular destination also increased one's probability of using a BIXI, but it was not as critical as proximity to home. Recreation-only cyclists and females were less likely to use a BIXI, whereas combining cycling and transit for trips and owning a driver's license made a person more likely to use BIXI. Although it may be counterintuitive that being a driver makes someone more likely to use shared bicycles, this result is consistent with the findings of a Chinese study (12). Age and distance from home to downtown would have a marginal but significant negative impact. The distance from home to downtown squared was also tested in this model to account for the possibility of a nonlinear relationship between distance and probability of use, but the variable was not significant and was thus removed from the final model.

TABLE 2 Factors Influencing Likelihood of Using Shared Bicycle Systems

\begin{tabular}{ll}
\hline Variable & Odds Ratio \\
\hline Age & $0.965^{* * *}$ \\
Gender (female) & $0.585^{* * *}$ \\
Owning a bicycle & $0.5778^{* *}$ \\
Cycling for recreational purposes only & $0.437^{* *}$ \\
Number of bicycle thefts & $1.104^{*}$ \\
Owning a driver's license & $1.588^{* *}$ \\
Annual household income from 0 to Can $\$ 40,000^{a}$ & $0.539^{* * *}$ \\
Being a bus user & $1.486^{* *}$ \\
Distance from home to downtown & $0.956^{* *}$ \\
Being a year-round cyclist & $0.539^{* * *}$ \\
Presence of a BIXI station less than $500 \mathrm{~m}$ from home & $3.245^{* * *}$ \\
Presence of a BIXI station less than $500 \mathrm{~m}$ from destination & $1.559^{* *}$ \\
Already combined cycling and transit & $1.772^{* * *}$ \\
\hline
\end{tabular}

NoTE: Constant $=0.769 ;-2 \log$ likelihood $=1,509.905 ;$ Nagelkerke $R^{2}=.241$. ${ }^{a}$ Can $\$ 1=\$ 1.02,2012$ U.S. dollars.

*Significant at the $90 \%$ confidence level; ** significant at the $95 \%$ confidence level; ***significant at the $99 \%$ confidence level. 
TABLE 3 Variables Influencing Frequency of Use of Shared Bicycle Systems per Cycling Season

\begin{tabular}{lc}
\hline Variable & Coefficient \\
\hline Constant & $9.766^{* *}$ \\
Age & -0.004 \\
Gender (female) & -1.335 \\
Owning a bicycle & $-5.680^{* *}$ \\
Owning a yearly BIXI membership & $15.911^{* * *}$ \\
Using BIXI to avoid risk of theft & $5.310^{* * *}$ \\
Using BIXI to avoid maintenance & $10.992^{* * *}$ \\
Using BIXI for its attractive design & $10.352^{* * *}$ \\
Number of bus stops within 400 m of home & $-0.093^{*}$ \\
Distance from home to downtown & $-1.142^{*}$ \\
Distance from home to downtown squared & $0.063^{* *}$ \\
Number of bicycle thefts & 0.455 \\
\hline
\end{tabular}

NotE: $N=535 ;$ adjusted $R^{2}=.502$.

*Significant at the $90 \%$ confidence level; **significant at the $95 \%$ confidence level; $* * *$ significant at the $99 \%$ confidence level.

People who earned less than Can $\$ 40,000(\mathrm{Can} \$ 1=\$ 1.02,2012$ U.S. dollars) per year were $32 \%$ less likely to be users of bicycle sharing than were people in other income brackets, corroborating findings of previous studies that users were mostly middle income; in Quebec, the median annual household income was slightly more than Can $\$ 64,000$ in $2009(12,13)$. People who cycled throughout the year and those who owned a bicycle were less inclined to use shared bicycles, which could mean that cycling enthusiasts were not typical BIXI users, who may have been more casual cyclists. Finally, each time an individual's bicycle was stolen increased that person's likelihood of being a BIXI user by $10 \%$.

Table 3 shows the findings from the linear regression that measured the number of times a person used the bicycle sharing system in the 2010 season. Some of the variables found in both models, such as age, gender, and number of bicycles stolen, were not significant for predicting the frequency of shared bicycle use, although they influenced the probability that someone would use shared bicycles. Ownership of a yearly BIXI membership had the greatest impact on the number of uses of shared bicycles, increasing by 15 the number of times a person would ride a BIXI per season. People who wanted to avoid maintenance of a bicycle and those who appreciated the design of the BIXI were likely to use it 10 more times per season. Bicycle ownership decreased the number of uses of a shared bicycle by 5.6 times per season. Living close to downtown slightly decreased the number of uses, although past a certain threshold, distance to downtown had the opposite effect. The presence of a BIXI station within a 500-m buffer at both ends of the trip was tested in the linear regression but proved to be insignificant and was removed from the final model. Finally, the number of bus stops within $400 \mathrm{~m}$ of home had a small but significant negative effect on frequency of use, which might indicate that BIXI was competing with transit.

\section{DISCUSSION OF RESULTS}

The most obvious finding of the first model is that a BIXI station close to home had the most influence on increasing the likelihood a rider would use shared bicycles. Proximity of the stations to regular destinations also augmented the odds a person would be a BIXI user, although the effect of this variable was not nearly as strong. This could in part be because only the most regular destination was included in the analysis. Therefore, to increase the modal share of BIXIs, more stations should be installed in residential neighborhoods in priority.

Docking stations were available only in central neighborhoods with good access to the transit network, and most users did not combine their BIXI ride with another mode for a single trip (probably because of good access to transit and proximity to downtown). It would be useful to observe patterns of use if BIXI stations were installed in peripheral neighborhoods or in areas with limited transit access. Many of the factors that increased the probability of use of shared bicycles were related to transportation habits; those persons combining cycling and transit, bus users, and people owning a driver's license were more likely to be BIXI users. Possession of a driver's license was expected to have the opposite effect, but the result was not as counterintuitive as it appears. Most adults in Montreal have a driver's license; therefore, chances were great that an adult user of shared bicycles also was a driver, whether they owned a car or drove on a regular basis.

Inversely, being a committed, year-round cyclist and owning a bicycle decreased one's chances of using BIXI. People who used their own bicycles had similar travel options and enjoyed the same health benefits as users of shared bicycle and were therefore not the group targeted by promotion campaigns for shared bicycle programs. However, it would be worthwhile to investigate whether bicycle owners choose not to use BIXI for convenience reasons or because of the cost of the membership, which could be prohibitive for someone who already has access to a personal bicycle free of charge (except maintenance).

An interesting finding from this study is that a person whose bicycle had been stolen was more likely to use shared bicycles. Respondents who expressed a concern about bicycle theft as a reason for using BIXI also rode shared bicycles more often. This indicates that BIXI is perceived as and acts as an effective solution for those who want to cycle yet are afraid of bicycle theft. The potential for shared bicycle systems to counteract the negative influence of bicycle theft on the modal share of cycling is obvious. This study goes one step further by confirming that this advantage was valued by individuals because it encouraged those who experienced theft to use the system and increased the frequency of use by individuals concerned about bicycle theft.

More generally, the results indicate that factors that prompt people to become shared bicycle users are not necessarily the same as those that increase the frequency of use. Spatial factors and transportation habits play an important role in encouraging individuals to use shared bicycles, yet specific respondent's motivations for using BIXIS have the greatest impact on the frequency of use.

The design of the BIXI caused individuals to use it on average 10 more times per year. This is a clear indication of the trendiness factor of the BIXI. The design of the BIXI and its promotion as an urban, environmentally friendly mode made use of shared bicycles trendy and consequently contributed to its popularity.

One limitation of the study is related to the distribution method of the survey and the risk of bias associated with voluntary-based surveys. This shortcoming was addressed through use of multiple dissemination tools to reach a broad cross section of the population. The study could use only socioeconomic, transportation habit, and spatial variables to generate the model; therefore the influence of respondent values could not be evaluated in the analysis. Tourists 
who used the system during visits were not included in the survey. A different study and a different approach are needed to capture the use of the BIXI system by this special population.

\section{CONCLUSION AND POLICY RECOMMENDATIONS}

A growing number of municipal and regional governments recognize and wish to enjoy the benefits of shared bicycle systems. A good understanding of factors that prompt or deter use of the system is necessary for the success of shared bicycle programs and for maximizing their potential. The presented study provided such an understanding of factors that influence the use of shared bicycle systems and those that affect frequency of use. The results of this research point to key elements for consideration in the formulation of policies that promote the use of shared bicycles. Interventions should focus on four major aspects:

1. The location of shared bicycle stations. The study showed that the location of docking stations was crucial for encouraging individuals to use shared bicycles. A greater number of docking stations close to the origins of potential users in residential neighborhoods is highly likely to generate an increase in the number of system users. The study also showed that proximity of docking stations to destinations augmented the odds of a person being a BIXI user, although its impact was smaller than that of proximity to origins.

2. The transportation habits of current and potential users. Transit users, people combining cycling and transit for their trips, and those who have a driver's license were more likely to use shared bicycle systems. Special multimodal offers, including access to shared bicycle systems, carsharing systems, or integrated multimodal fare cards, would encourage individuals to adopt shared bicycles by making integration into their current travel habits as seamless as possible.

3. The fear of bicycle theft. The study confirmed that individuals recognized shared bicycles as an active travel option that minimized bicycle theft. Promotion of the shared bicycle systems should emphasize this advantage to attract new users and increase frequency of use among those whose bicycles have been stolen or those concerned about theft.

4. The status and perceptions associated with shared bicycles. Individuals who liked the design of shared bicycles tended to use the system more often. Advertising campaigns that convey that use of shared bicycles is trendy are likely to encourage users to increase their frequency of use.

This research, based on a survey of people living in the Montreal region, provided findings that are consistent with results of previous studies conducted elsewhere in the world. Although each region has its particularities, the main findings of this study could be useful for any city wanting to maximize the potential of its shared bicycle system.

In the case of Montreal, implementation of the BIXI system had effects that have gone beyond increased use of shared bicycles. Many experts have observed an increase in the overall number of cyclists and a positive shift in social status of utilitarian cycling in general. In this case, the implementation of a shared bicycle system in the city not only improved the range of sustainable travel options but also contributed to the cycling culture in Montreal, an effect that requires further investigation in the near future.

\section{ACKNOWLEDGMENTS}

The authors thank the dedicated transit users and cyclists who participated in the 2010 TRAM survey. The authors acknowledge the feedback and comments provided on the questionnaire by the members of the TRAM research group and thank Jacob Larsen, who helped in the design of the survey, as well as Lise Gauvin and Daniel Fuller of the University of Montreal. The authors also thank the three reviewers for their feedback.

\section{REFERENCES}

1. Yang, L., S. Sahlqvist, A. McMinn, S. Griffin, and D. Ogilvie. Interventions to Promote Cycling: Systematic Review. British Medical Journal, Vol. 341, 2010, p. C5293.

2. Shaheen, S. A., S. Guzman, and H. Zhang. Bikesharing in Europe, the Americas, and Asia: Past, Present and Future. In Transportation Research Record: Journal of the Transportation Research Board, No. 2143, Transportation Research Board of the National Academies, Washington, D.C., 2010, pp. 159-167.

3. DeMaio, P. Bike-Sharing: History, Impacts, Models of Provision, and Future. Journal of Public Transportation, Vol. 12, No. 4, 2009, pp. 41-56.

4. Gradinger, K. The Evolution of Bike Sharing Programs. Bike Share Philadelphia, 2007. http://bikesharephiladelphia.org/learn/history. Accessed April 11, 2011.

5. Krykewycz, G. R., C. M. Puchalsky, J. Rocks, B. Bonnette, and F. Jaskiewicz. Defining Primary Market and Estimating Demand for Major Bicycle-Sharing Program in Philadelphia, Pennsylvania. In Transportation Research Record: Journal of the Transportation Research Board, No. 2143, Transportation Research Board of the National Academies, Washington, D.C., 2010, pp. 117-124.

6. Morency, C., M. Trépanier, and F. Godefroy. Insight into Montreal's Bikesharing System. Presented at 90th Annual Meeting of the Transportation Research Board, Washington, D.C., 2011

7. Enquête Origine-Destination. L'Agence Métropolitaine de Transport, Montreal, Quebec, Canada, 2008

8. Fuller, D. Physical Activity Levels Related to an Urban Bicycle Sharing Program: BIXI Montreal. Alberta Centre for Active Living, Edmonton, Alberta, Canada, 2010.

9. Bixi Système. 2010. http://www.bixisysteme.com/systeme_bixi/le_ velo. Accessed March 17, 2011.

10. Bixi Montréal. 2010. http://montreal.bixi.com/nouvelles/categorie/ BIXI\%20en\%20chiffres. Accessed March 15, 2011.

11. Dillman, D., J. Smyth, and L. Christian. Internet, Mail, and MixedMode Surveys: The Tailored Design Method, 3rd ed. John Wiley \& Sons, Hoboken, N.J., 2009.

12. Shaheen, S. A., H. Zhang, E. Martin, and S. Guzman. China's Hangzhou Public Bicycle: Understanding Early Adoption and Behavioral Response to Bikesharing. In Transportation Research Record: Journal of the Transportation Research Board, No. 2247, Transportation Research Board of the National Academies, Washington, D.C., 2011, pp. 33-41.

13. Statistics Canada. 2007. http://www40.statcan.ca/101/cst01/famil108a-eng htm. Accessed Oct. 8, 2011.

The Bicycle Transportation Committee peer-reviewed this paper. 